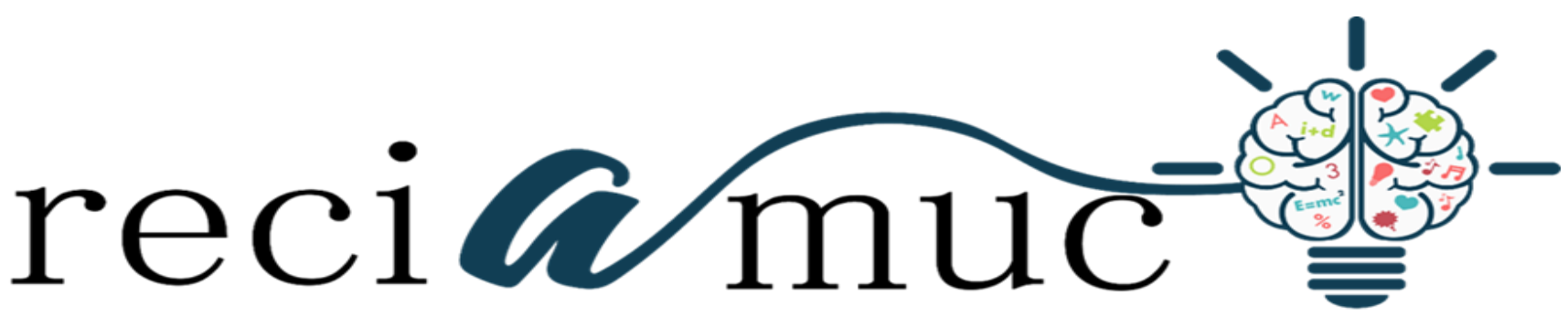

Revista cientifica de investigación actualización del mundo de las ciencias

Francisco Xavier Hugo Cárdenas ${ }^{\text {a }}$; Cesar Eduardo Jiménez Rosero b; Carlos Alberto Vasco Gualotuña ${ }^{c}$; José Luis Saa Loor ${ }^{d}$

La RSC en las empresas una estrategia rentable que necesita de impulsos legales

CSR in companies a profitable strategy that needs legal impulses

Revista Científica de Investigación actualización del mundo de las Ciencias. Vol. 3 núm., 1, enero, ISSN: 2588-0748, 2018, pp. 353-376

DOI: $10.26820 /$ reciamuc/3.(1).enero.2019.353-376

URL: http://reciamuc.com/index.php/RECIAMUC/article/view/239

Código UNESCO: 5311 Organización y Dirección de Empresas

Tipo de Investigación: Artículo de Revisión

Editorial Saberes del Conocimiento

Recibido: 10/12/2018

Aceptado: 20/01/2019

Publicado: 30/01/2019

Correspondencia: director@ reciamuc.com

a. Magister Seguridad Salud y Ambiente; Ingeniero Ambiental.

b. Magister en Seguridad y Salud Ocupacional; Ingeniero en Maderas.

c. Magister en Seguridad y Salud Ocupacional; Ingeniero en Maderas.

d. Magister Seguridad Salud y Ambiente; Ingeniero Químico Especialización: Ingeniero Químico. 


\section{La RSC en las empresas una estrategia rentable que necesita de impulsos legales}

Vol. 3, núm. 1., (2019)

Francisco Xavier Hugo Cárdenas; Cesar Eduardo Jiménez Rosero; Carlos Alberto Vasco Gualotuña; José Luis Saa Loor

\section{RESUMEN}

Las empresas a nivel mundial para mantenerse dentro de un contexto competitivo, tienen la responsabilidad de buscar nuevas acciones dirigidas a mejorar sus interacciones con el entorno inmediato, es decir, dejar a un lado su visión centrada en obtener resultados económicos como pilar fundamental, requieren cambiar su postura empresarial por convertirse en agentes que propicien transformaciones no sólo en lo interno, sino en la sociedad misma. Desde esta perspectiva, pueden competir sanamente para afianzar una modificación permanente en las áreas sociales, ambientales y del medio donde se circunscribe. No obstante, Cortina (2014) indica “todas las empresas significan una amenaza no sólo para la sociedad y el medio ambiente, sino para sí mismo, pues carecen de sostenibilidad y su competitividad las obligan a buscar constantemente estrategias que le permitan logra sus objetivos".(p.36). Es claro entender, según el planteamiento la necesidad que poseen las empresas para responder positivamente a sus objetivos, de un marco legal encargado de llevarlas a cumplir no sólo sus obligaciones con las demás organizaciones, sino elevar la competitividad interna donde el talento humano reciba una adecuada atención enmarcada en aquellos lineamientos jurídicos que garanticen sus beneficios laborales y al mismo tiempo hagan posible alcanzar el crecimiento organizacional desde el contexto de convertirse en empresarios que implementen estrategias cónsonas a sus propias necesidades para tener eficiencia en las tareas propuestas independientemente de cuales sean. Estas ideas, direccionan el contenido del artículo que tiene como objetivo analizar la RSC en las empresas una estrategia rentable que necesita de impulsos legales, bajo una visión metodológica documental, caracterizada por técnicas centradas en la lectura interpretativa para finalmente exponer importantes valoraciones generales.

Palabras Claves: RSC; Empresas; Estrategia Rentable; Impulsos Legales. 


\title{
La RSC en las empresas una estrategia rentable que necesita de impulsos \\ legales
}

Vol. 3, núm. 1., (2019)

Francisco Xavier Hugo Cárdenas; Cesar Eduardo Jiménez Rosero; Carlos Alberto Vasco

Gualotuña; José Luis Saa Loor

\begin{abstract}
Companies worldwide to stay within a competitive context, have the responsibility to seek new actions aimed at improving their interactions with the immediate environment, that is, leaving aside their vision focused on obtaining economic results as a fundamental pillar, they need to change his business position to become agents that promote transformations not only internally, but in society itself. From this perspective, they can compete healthily to secure a permanent modification in the social, environmental and environmental areas where it is circumscribed. However, Cortina (2014) indicates that "all companies represent a threat not only to society and the environment, but to themselves, because they lack sustainability and their competitiveness forces them to constantly search for strategies that allow them to achieve their objectives". (p.36). It is clear to understand, according to the approach, the need that companies have to respond positively to their objectives, of a legal framework in charge of carrying them to fulfill not only their obligations with other organizations, but to increase internal competitiveness where human talent receives an adequate attention framed in those legal guidelines that guarantee their labor benefits and at the same time make it possible to achieve organizational growth from the context of becoming entrepreneurs that implement strategies consonant with their own needs to be efficient in the proposed tasks regardless of what they are. These ideas, direct the content of the article that aims to analyze CSR in companies a profitable strategy that needs legal impulses, under a documentary methodological vision, characterized by techniques focused on interpretative reading to finally expose important general assessments.
\end{abstract}

Key Words: CSR; Companies; Profitable Strategy; Legal Impulses. 


\section{La RSC en las empresas una estrategia rentable que necesita de impulsos legales}

Vol. 3, núm. 1., (2019)

Francisco Xavier Hugo Cárdenas; Cesar Eduardo Jiménez Rosero; Carlos Alberto Vasco Gualotuña; José Luis Saa Loor

\section{Introducción.}

El crecimiento económico agregado en las sociedades avanzadas, se convierte en un agente que obliga a las empresas a mantener criterios claros en función a sus objetivos y obligaciones directas e indirectas con el medio donde se circunscribe, es decir, debe mejorar su visión hacia las comunidades, mediante la búsqueda de estrategias viables encargadas de asegurarle su competitividad como respuesta directa ante los procesos de transformación requeridos en un momento determinado. Esto permite, aclarar que hoy las empresas como organizaciones incursas en un espacio comunitario, debe redimensionar sus relaciones con la sociedad a fin de no sólo buscar valores para sus miembros, sino asumir sus responsabilidades ante los grupos de interés.

De acuerdo con lo anterior, cada empresa para mantener su competitividad, debe implementar estrategias encargadas de ayudarlas a responder con eficacia ante sus metas colectivas, mediante el cumplimiento normado por los reglamentos jurídicos que hacen posible atender sus necesidades. En otras palabras, es fundamental el emplear políticas básicas que hagan posible agregar en sus diferentes espacios la responsabilidad social corporativa, como una forma general que promueva un cambio progresivo en las operaciones empresariales y no limitarse a acciones aisladas; todo lo contrario buscar mecanismos internos que le ayuden a lograr reciprocidad entre todos sus miembros.

Desde esta perspectiva, la Responsabilidad Social Corporativa conduce a las empresas a que se conviertan en el eje del sistema productivo, esto hace posible asumir compromisos para lograr una convivencia en armonía entre sus actividades lícitas dirigidas a obtener beneficios y 


\section{La RSC en las empresas una estrategia rentable que necesita de impulsos \\ legales}

Vol. 3, núm. 1., (2019)

Francisco Xavier Hugo Cárdenas; Cesar Eduardo Jiménez Rosero; Carlos Alberto Vasco

Gualotuña; José Luis Saa Loor

crear valor para sus socios y participes, es decir, plantearse objetivos globales de la sociedad que introduzca la sostenibilidad del crecimiento económico a largo plazo en una necesaria conservación de los recursos naturales que aseguren un comportamiento ético funcional para la empresa con su entorno.

$\mathrm{Al}$ respecto, Ibañez (2014) destaca lo siguiente:

Mediante la responsabilidad social corporativa se logran integrar estrategias, generar voluntariedad más allá de la legislación, el cumplimiento de la ley es sólo su inicio, sus acciones son siempre para atender expectativas o necesidades de grupos de interés, nunca para exclusivo beneficio propio y la difusión debe ser comunicada y sostenerse al escrutiño de stakeholders de la empresa. (p. 3)

Lo citado, permite valorar que la aplicación de la responsabilidad social corporativa como estrategia, le asegura a los gerentes una dinámica centrada en revisar de forma permanente los objetivos trazados, pues, no sólo buscar la norma jurídica como base determinante del hacer empresarial, sino como un estímulo encargado de elevar sus opciones gerenciales en pro de garantizar el inicio de sus acciones, ajustadas a las expectativas previamente establecidas o necesidades, de allí, que su inclusión como herramienta hace posible crear condiciones viables para ofrecer resultados positivos a todos sus miembros y al mismo tiempo mediante niveles comunicacionales efectivos abrir un compás de informaciones entre los grupos para deliberar las alternativas que se tomarán y que abrirán a la empresa hacia la sociedad en general. 


\section{La RSC en las empresas una estrategia rentable que necesita de impulsos legales}

Vol. 3, núm. 1., (2019)

Francisco Xavier Hugo Cárdenas; Cesar Eduardo Jiménez Rosero; Carlos Alberto Vasco Gualotuña; José Luis Saa Loor

En este mismo contenido temático, Zavala (2015) define la responsabilidad social corporativa como:

Su impacto en la sociedad, lo que implica que las organizaciones empresariales deben integrar las preocupaciones sociales, medioambientales y éticas; respetar los derechos humanos; y tener en cuenta las preocupaciones de los consumidores. Así crean valor, pudiendo identificar, prevenir y atenuar las posibles consecuencias adversas. (p.2)

La visión proporcionada, lleva a reflexionar que mediante la introducción de la responsabilidad social corporativa, cada empresa no sólo alcanzar sus objetivos y beneficios económicos- financieros, sino que debe mirar, su entorno como parte esencial para darle continuidad a los objetivos trazados, valorar la necesidad de reconocer ese espacio como un medio determinante en sus relaciones internas y así fortalecer la construcción de la honestidad como valor esencial que le dará cabida a identificar aquellas posibles situaciones adversas para su posterior interpretación e introducción de acciones innovadoras competentes que darán respuesta favorable durante la realización de su tareas empresariales.

En consecuencia, cada empresa al buscar cambios progresivos en la organización y sus actividades, debe estimar la presencia de aquellos grupos de interés que son determinantes al momento de llegar a tomar decisiones y la ejecución de las mismas, es decir, incluir en el trabajo a los accionistas, clientes, proveedores, administración, sociedades, entidades financieras entre otros, en definitiva, todos aquellos que establecen o dictan principios a la organización para así cumplir los objetivos. De este modo, se comprende que la gestión empresarial responsable debe 


\section{La RSC en las empresas una estrategia rentable que necesita de impulsos \\ legales}

Vol. 3, núm. 1., (2019)

Francisco Xavier Hugo Cárdenas; Cesar Eduardo Jiménez Rosero; Carlos Alberto Vasco

Gualotuña; José Luis Saa Loor

estar fundamentada en valores como: transparencia, honestidad y ética, normas sociales que le darán posibilidades de mejorar las condiciones del entorno, ayudan a la identificación y reducción de los riesgos para así posible el fortalecimiento de su reputación llegando a aumentar la rentabilidad.

Todo lo anterior, lleva a entender que mediante la incorporación de la responsabilidad social corporativa, las empresas inducen a tener efectivos positivos a medio y largo plazo; además contribuye a aumentar el rendimiento, así como el valor de las mismas para el total de los grupos e interés. Por lo tanto, su determinación debe estar sujeta a las ordenamientos legales presente en cada sociedad, mediante ellos puede asegurar no sólo resultados viables para su estructura organizativa, sino, que hace posible agregar la introducción de acciones beneficiosas para todos los actores sociales, es decir, cada Estado como garante del cumplimiento de las leyes que norman la vida empresarial, busca mediante una vigilancia permanente implementar controles básicos que garanticen su crecimiento global.

Al continuar con el análisis correspondiente, se puede precisar que mediante un manejo efectivo de la responsabilidad social corporativa, cada empresa tiene posibilidades únicas de llevar adelante un negocio rentable; pues, la misma tiene en cuenta todos los efectos ambientales, sociales y económicos tanto positivos como negativos que se generan en la sociedad. Esto hace posible dar respuestas oportunas que buscan simplemente las obligaciones correspondiente organización, para así diferenciarse entre ellas, pues, estas actividades vinculadas con la responsabilidad social como estrategia, generan ventajas competitivas y contribuyen a asegurar una separación entre las diferentes empresas, dado que, la inclusión de la inversión social, 


\section{La RSC en las empresas una estrategia rentable que necesita de impulsos legales}

Vol. 3, núm. 1., (2019)

Francisco Xavier Hugo Cárdenas; Cesar Eduardo Jiménez Rosero; Carlos Alberto Vasco Gualotuña; José Luis Saa Loor

simplemente agrega cambios básicos en el mejoramiento de las condiciones de vida de la comunidad e incrementar la reputación de la empresa, en otras palabras, se construye un verdadero cumplimiento de las leyes emanadas por la sociedad.

Cabe agregar que, al vincular estas consideraciones con las empresas latinoamericanas, se puede encontrar que las mismas para darle cabida a la responsabilidad social corporativa, debe mirar las nuevas concepciones que el Estado ha construido en materia del pensamiento empresarial; es decir, agregar en sus estructuras, objetivos, metas y propuestas el humanismo como principio básico que valora la existencia del individuo en un entorno, tal como lo aseveran Villacís, Suárez y Guillin, (2016)

En los países latinoamericanos se han realizado cambios en cuestión de legislación y exigencias a las empresas, haciendo que el sector privado se preocupe cada vez más por los efectos de sus actos en todos los ámbitos mencionados. Se han elegido el humanismo contemporáneo y la ética empresarial y ética ecológica como corrientes de pensamiento. (p.454)

De acuerdo con estos planteamientos, se puede decir que las empresas a nivel latinoamericano, han busca mirar la estructura interna que caracteriza a las organizaciones y pro de ello, emerge un nuevo concepto del pensamiento empresarial, al concebir la práctica gerencial en las organizaciones, desde la presencia del enfoque humanístico como principio que busca no sólo entender el comportamiento humano, sino valorar las diferentes situaciones que se registran en su entorno como resultado de las relaciones llevadas a cabo. Esto conduce a precisar que, las empresas deben concebir el trabajo desde la perspectiva de comprender el ser como base de su 


\section{La RSC en las empresas una estrategia rentable que necesita de impulsos \\ legales}

Vol. 3, núm. 1., (2019)

Francisco Xavier Hugo Cárdenas; Cesar Eduardo Jiménez Rosero; Carlos Alberto Vasco

Gualotuña; José Luis Saa Loor

ética empresarial, para ello, norma las actividades en función a las leyes que fija el país para obtener controles y cumplimiento de aquellas reglas previamente establecidas.

No obstante, los tiempos actuales como resultado a la presencia de crisis mundiales se convierten en actores esenciales para negar las posibilidades de mantener una verdadera conexión en el cumplimiento de la responsabilidad social corporativa, las empresas buscan sólo generar beneficios económicos y financieros, negando posibles actuaciones positivas hacia el recurso humano y el entorno social. En otras palabras, permanecen distante de las realidades presente en su comunidad, tal como lo argumenta, Padilla (2017)

En los tiempos de crisis las empresas tienden a olvidarse de la responsabilidad social corporativa, prima lo urgente sobre lo importante, piensan en la rentabilidad y sobre todo en sobrevivir, reducen las inversiones de personal, bajada de sueldos entre otros aspectos, e incluso toman decisiones que conllevan incumplimiento legales con las que pretenden reducir costos. (p. 15)

Al responder a las ideas citadas por el autor, se puede entender que existe una necesitada tangible, de recurrir de forma directa y precisa al reconocimiento de los ordenamientos legales como normas encargadas de exigir a las diferentes empresas el cumplimiento de la responsabilidad social corporativa, de lo contrario los trabajadores son dejados a un lado, carecen de interés como beneficiados sociales, simplemente se plantean medidas que van dirigidas directamente a lograr el crecimiento económico de la empresa, se olvidan del significado que 


\section{La RSC en las empresas una estrategia rentable que necesita de impulsos legales}

Vol. 3, núm. 1., (2019)

Francisco Xavier Hugo Cárdenas; Cesar Eduardo Jiménez Rosero; Carlos Alberto Vasco Gualotuña; José Luis Saa Loor

poseen la teoría del humanismo como nuevo pensamiento en las empresas modernas. Es allí, donde cada sociedad a través de impulsos jurídicos debe crear una visión legal encargada de norma las actividades empresariales como una forma de mantener en sus estructuras la presencia de beneficios colectivos.

Cada uno de estos últimos argumentos, llevan a encontrar que reviste de importancia la presencia de estímulos legales encargados de direccionar la responsabilidad social corporativa hacia una nueva visión integral, donde todos los involucrados en la práctica empresarial, sean vistos como medios fundamentales para lograr objetivos a corto, mediano y largo plazo, de esta manera, se podrá hablar en el tiempo de mejoras continuas, beneficios colectivos a los trabajadores, como la satisfacción personal y cambios inminentes en la comunidad.

\section{Método.}

Para mantener la operatividad metodológica del presente artículo, se determina la selección del método como herramienta esencial encargada de proporcionar visiones generales en relación al contenido tratado, para lo cual, es importante considerar la definición dada por, Ventura (2018) los métodos de investigación "son aquellos que le aseguran al investigador la obtención del conocimiento desde una visión global de los hechos".(p.69). Por lo tanto, la escogencia del camino para reflexionar en cuanto a los diferentes tópicos esenciales que dan cabida a la construcción de un cuerpo interpretativo y de apoyo documental para este proceso investigativo, se estima la selección del inductivo. 


\section{La RSC en las empresas una estrategia rentable que necesita de impulsos \\ legales}

Vol. 3, núm. 1., (2019)

Francisco Xavier Hugo Cárdenas; Cesar Eduardo Jiménez Rosero; Carlos Alberto Vasco

Gualotuña; José Luis Saa Loor

Al establecer como método el tipo inductivo, significa que la indagación de los distintos contenidos fueron valorados desde una visión individual, para luego conjugarlos y trasladar las interpretaciones a contexto global, de esta forma se diseñan las apreciaciones enmarcadas en aquellas situaciones que forman parte del fenómeno en estudio. En tal sentido, Ventura (ob.cit) destaca que el método inductivo "lleva a leer, interpretar, separar y complementar con las experiencias del investigador para generar nuevas visiones vinculadas con un tema previamente seleccionado". (p.71).

Tipo de Investigación

La continuidad del proceso investigativo, permite tener el respectivo acercamiento a la identificación de su tipo en correspondencia con un nivel que según Arias (2012) "se refiere al grado de profundidad con que se aborda un objeto o fenómeno" (p.45). Por lo tanto, el desarrollo de esta actividad, lleva a resaltar que debido a estar en correspondencia con el tipo documental, su contenido estará estimado por un nivel donde se logra no sólo interpretar los argumentos básicos del fenómeno, sino que se amplían sus valoraciones para así generar nuevas valoraciones significativas e importantes para otra actividad investigativa

En función a lo anterior, se puede indicar que toda investigación documental de acuerdo con Sandoval (2015) "representa el estudio de un problema con el propósito de ampliar y profundizar el conocimiento de su naturaleza con apoyo en trabajos previos divulgados por medios impresos o electrónicos" (p.60). De allí, que se estimaron una serie de actividades centradas en lograr recabar las informaciones previas que dieron como resultado, la respectiva elaboración del análisis relacionado con el fenómeno en estudio. 


\section{La RSC en las empresas una estrategia rentable que necesita de impulsos legales}

Vol. 3, núm. 1., (2019)

Francisco Xavier Hugo Cárdenas; Cesar Eduardo Jiménez Rosero; Carlos Alberto Vasco Gualotuña; José Luis Saa Loor

\section{Fuentes Documentales}

Cada una de las consideraciones expuestas en párrafos anteriores, permiten comprender que la conducción efectiva del proceso investigativo, necesita la presencia de herramientas básicas que ayuden a la selección adecuada de los aspectos relativos al fenómeno en estudio, para ello, se incorporaron aquellos medios esenciales que dan cabida a la organización del texto. En esta dirección Sandoval (ob.cit) las define "como la suma de medios especiales que están directamente vinculados con el tipo de investigación y se incorporar para recopilar la información previa" (p.65). En consecuencia, para el desarrollo del artículo, se tuvo que adecuar las herramientas de lecturas rápida, reflexiva, el fichaje, organización de textos entre otras fuentes documentales.

\section{Técnicas para la Recolección de la Información}

El desarrollo del contenido que caracteriza a este artículo, se encuentra determinado por la necesidad de incluir en su realización diferentes técnicas viables que dieron como resultado una recopilación importante y de interés para dar continuidad al fenómeno a indagar, tal como lo expresa Sandoval (ob.cit) las técnicas de recolección de información "sirven de apoyo para la verificación de un contenido en particular y hace posible reconocer los diferentes elementos que lo representan en un momento determinado".(p.66). Por ello, se incorporó la lectura rápida mediante la cual, se pudo hacer una arqueo de las referencias más importantes para ampliar los contenidos seleccionados, asimismo, se llevó a cabo el fichaje mediante el cual, se pudo estimar la realidad de las apreciaciones dadas por los teóricos desde una perspectiva global y construir 


\section{La RSC en las empresas una estrategia rentable que necesita de impulsos \\ legales}

Vol. 3, núm. 1., (2019)

Francisco Xavier Hugo Cárdenas; Cesar Eduardo Jiménez Rosero; Carlos Alberto Vasco

Gualotuña; José Luis Saa Loor

definitivamente los argumentos básicos que dieron cabida a la ampliación del contenido propuesto.

\section{Resultados.}

A continuación se desglosan una serie de interpretaciones encargadas de ofrecer al lector una visión general del tema, fijar posiciones especiales o particulares que hagan entender la significación que posee la responsabilidad social corporativa en el marco empresarial, desde un apoyo legal para cubrir no sólo necesidades económicas, sino buscar de forma directa el mejoramiento continuo que el talento humano debe recibir dentro de los espacios organizacionales y su relación con el entorno circundante.

Conocimiento Empresarial desde una Visión de la Responsabilidad Social

Actualmente, las empresas se encuentran en medio de condiciones adversas, caracterizadas por la sobreoferta de productos, servicios y altos niveles de incertidumbre; estas u otras manifestaciones externas las han llevado a dedicar esfuerzo administrativo hacia la búsqueda de nuevas alternativas que permitan contrarrestar los efectos de la competencia, debido a los drásticos cambios comerciales a nivel mundial. En este panorama, las empresas intentan desarrollar e implementar políticas o estrategias que les ayuden a crecer para consolidarse. Las 


\section{La RSC en las empresas una estrategia rentable que necesita de impulsos legales}

Vol. 3, núm. 1., (2019)

Francisco Xavier Hugo Cárdenas; Cesar Eduardo Jiménez Rosero; Carlos Alberto Vasco Gualotuña; José Luis Saa Loor

estrategias que involucran la Responsabilidad Social están al orden del día y surgen, entonces, como una expresión manifiesta de las nuevas exigencias de los grupos de interés (stakeholders).

De acuerdo a las ideas anteriores, es conocido que el crecimiento empresarial es uno propósito incuestionable de las organizaciones. Según Blanco (2014) define el conocimiento empresarial como "un objetivo inherente; a las organizaciones que buscan crecer, con el fin de obtener un beneficio que les permita ser sostenibles" (p.36). Es decir, se requiere crear una imagen corporativa positiva para implementar el bienestar institucional, visión que le dará oportunidad para garantizar su permanencia y consolidar el ámbito empresarial.

Cabe agregar, que las empresas en los últimos la empresa en los últimos años juega un rol diferente y ha cambiado la concepción que se tiene de ella, pues, se ha convertido en un factor de cambio e influencia social. Lo que las empresas hacen o dejan de hacer o afecta de manera significativa su entorno y a su ámbito más directo. Es decir, existe una conciencia de la importancia de la empresa en la sociedad y del poder que ella tiene para ser actor de cambio; esto hace posible generar un mercado competitivo, debido a exigir respuestas que lleven a las empresas a ser responsables, acepten y cumplan sus obligaciones con los trabajadores y el entorno social.

Por lo tanto, reconocer la empresa desde una perspectiva diferente, donde el conocimiento empresarial sea la fuente básica para modificar aquellos problemas e introducir nuevas acciones encargadas de transformar sus estructuras internas como representación de conocer y diagnosticar la realidad circundante. Al respecto, Bank (2014) dice que "reconocer esta nueva perspectiva de la empresa en cuanto a su poder y responsabilidad social sin dejar de 


\section{La RSC en las empresas una estrategia rentable que necesita de impulsos \\ legales}

Vol. 3, núm. 1., (2019)

Francisco Xavier Hugo Cárdenas; Cesar Eduardo Jiménez Rosero; Carlos Alberto Vasco

Gualotuña; José Luis Saa Loor

lado su crecimiento financiero le permite encontrar un enfoque para los problemas sociales".

(p.125). Lo citado, lleva a entender que mediante la incorporación del conocimiento empresarial, los gerentes tienen oportunidad de agregar nuevas estrategias de cambio, donde cada actor sea un ente involucrado para responder a los objetivos trazados, sin generar significación predeterminada por lo económico, sino todo lo contrario mirar la empresa como un todo que al ser analizada se descubren los factores externos e internos para resolver mediante estrategias sociales significativas.

En consecuencia, el reconocimiento social que hacen los administradores de la empresa asegura con anticipación que tienden a manejar relaciones con la sociedad, no desde una perspectiva abstracta, sino desde múltiples grupos de interés donde esta actividad es directa, relevante, real y manejable. De allí, que los llamados grupos de interés van a comprender los empleados, accionistas, clientes, proveedores aunado a los grupos de interés público. Para Bank (ob.cit) considera que los grupos de interés "se clasifican en internos donde se encuentran los directivos así como los trabajadores, además de los externos que hacen vida en la comunidad". (p. 135). Esto lleva a entender que las empresas para propiciar cambios progresivos con la sociedad, debe no sólo identificar aquellos grupos de interés que forman parte de ella; sino concebir la práctica empresarial desde la existencia de una comunidad y así fijar una verdadera vinculación entre las partes.

Según este último autor, precisa que para lograr una verdadera interpretación del conocimiento empresarial bajo la visión de la responsabilidad social es importante que cada departamento empresarial, tome el significado que posee para así generar utilidad y vigilar el 


\section{La RSC en las empresas una estrategia rentable que necesita de impulsos legales}

Vol. 3, núm. 1., (2019)

Francisco Xavier Hugo Cárdenas; Cesar Eduardo Jiménez Rosero; Carlos Alberto Vasco Gualotuña; José Luis Saa Loor

comportamiento económico, la misión organizativa, su propósito corporativo y los códigos de la empresa para tener cabida a una permanencia. Asimismo, es importante entender que la presencia de los accionistas permite a la gerencia garantizar la transparencia y sus derechos mediante el establecimiento y cumplimiento de reglas justas y concertadas. De igual manera, es importante mirar a los empleados o colaboradores: el objetivo con este grupo es proveerlos de las herramientas que hagan posible su desarrollo profesional y personal, garantizándoles así una mejor calidad de vida, reflejada en las compensaciones, recompensas, programas de salud y asistencia, licencias, despidos, apelaciones, renuncias, paros, discriminación, acomodo de familia, seguridad, planeación de carrera, entre otros. Los asuntos de los clientes, vistos desde la perspectiva de la Responsabilidad Social buscan satisfacer sus necesidades entregándoles productos o servicios de alta calidad.

En razón de las ideas anteriores, se puede entender que la responsabilidad social hace posible afianzar el crecimiento empresarial visto como un proceso intangible que depende de elementos tangibles (acumulación de capital físico y humano), y sobre todo de una adecuada organización y estructura interna, el crecimiento se caracteriza por la acción compleja de las modificaciones internas que desembocan en un aumento de las dimensiones y cambios en las características de los objetos sometidos a tal proceso. En el caso de las empresas, la acumulación de capital físico y humano y el aumento en las dimensiones reflejan cambios que se evidencian después de una mayor aceptación de los productos y/o servicios de la organización en el entorno; mediante la expansión que realiza la empresa hacia nuevos mercados, tanto geográficos como de clientela. Las compañías en crecimiento se expanden en nuevos mercados y segmentos de 


\section{La RSC en las empresas una estrategia rentable que necesita de impulsos \\ legales}

Vol. 3, núm. 1., (2019)

Francisco Xavier Hugo Cárdenas; Cesar Eduardo Jiménez Rosero; Carlos Alberto Vasco

Gualotuña; José Luis Saa Loor

mercado, nuevas regiones, incluso nuevos países. Esto hace posible lograr el respectivo crecimiento de una empresa logre conseguir a los mejores empleados, conserve, como es bien sabido, las capacidades, habilidades y competencias del personal se podrían constituir en fuente de ventaja competitiva.

\section{La Responsabilidad Social Corporativa como Estrategia para el Crecimiento Empresarial}

Ante los nuevos cambios introducidos en la visión social que tienen hoy las empresas, se encuentra que la responsabilidad social corporativa se visualiza como una estrategia que debe ser vista desde un contexto jurídico para asegurar el alcance de beneficios colectivos, por cada nación; es decir, es importante ofrecer a las empresas basamentos legales que al ser implementados en la vida empresarial, les permita propiciar los cambios externos como internos y así, dirigir nuevos caminos hacia su crecimiento integral.

Según Baldillo (2017) "los cambios globalizados han hecho que las empresas conciba la responsabilidad social corporativa como una estrategia que incrementa su rentabilidad". (p.51). Al considerar esta apreciación, se puede decir que mediante la utilización de la responsabilidad social corporativa, cada empresa logra introducir diferentes estrategias viables que aseguran la introducción de nuevas perspectivas en la comunidad e interno. Asimismo, el autor citado, precisa una serie de beneficios agregados a las empresas al utilizar la responsabilidad social corporativa, entre los cuales destaca los siguientes:

Beneficios para los grupos de interés: El compromiso que asume la empresa con la Responsabilidad Social arroja beneficios para los grupos de interés involucrados, aumento en las 


\section{La RSC en las empresas una estrategia rentable que necesita de impulsos legales}

Vol. 3, núm. 1., (2019)

Francisco Xavier Hugo Cárdenas; Cesar Eduardo Jiménez Rosero; Carlos Alberto Vasco Gualotuña; José Luis Saa Loor

ventas, incremento en las utilidades, ampliación de la capacidad de planta, apoyo a la formación de los empleados, generación de nuevos empleos, inversión social (parques, escuelas, jornadas de salud, jornadas de capacitación, por mencionar algunas), convenios ampliados con los proveedores; todo ello en estrecha relación con la construcción de tejido social.

La reputación de la empresa: Puede considerarse el resultado de un proceso competitivo en el cual las empresas transmiten sus características clave a sus grupos de poder stakeholders, para maximizar sus estatus moral y socio económico. En este sentido, muestran los componentes de la reputación, para así facilitar las prácticas de liderazgo global que logren conseguirla: reputación por la confianza entre los empleados a través de prácticas de gestión ejemplares; reputación por credibilidad de inversores, mostrando la rentabilidad para los accionistas y manteniendo resultados estables y promoviendo el crecimiento financiero; reputación por la confianza entre clientes y proveedores asegurando calidad, servicio e innovación; reputación por la responsabilidad entre la comunidad y los entes públicos; reputación por la contabilidad entre el Gobierno y los intereses de los competidores.

Es decir, con la Responsabilidad Social Corporativa se aumenta la buena reputación de la empresa, la cual, a su vez, la convierte en empleadora más atractiva y, por lo tanto, atrae a más solicitantes del mercado laboral. De esta manera, la Responsabilidad Social Corporativa puede brindar ventajas competitivas, pues ayuda a atraer y conservar empleados de calidad superior.

Las acciones socialmente responsables: Los problemas de la sociedad pueden representar oportunidades de negocio, además de que es posible obtener utilidades mediante esfuerzos sistemáticos y vigorosos para resolver estos problemas. En otras palabras, conviene 


\section{La RSC en las empresas una estrategia rentable que necesita de impulsos \\ legales}

Vol. 3, núm. 1., (2019)

Francisco Xavier Hugo Cárdenas; Cesar Eduardo Jiménez Rosero; Carlos Alberto Vasco

Gualotuña; José Luis Saa Loor

actuar éticamente, ya que esto redunda en beneficios para la organización a mediano y largo plazo. Realizar obras de gran alcance e impacto social permite que la organización se posicione con mayor facilidad en las mentes de sus grupos de interés.

La honestidad y justicia: Incluidos el reconocimiento de los errores, las disculpas genuinas, rápidas, sinceras, enmendar errores pueden generarle grandes ganancias a la reputación personal e imagen pública de la compañía al dar respuesta de los grupos de interés; será siempre positiva cuando se evidencia deseos o actuaciones sinceras que lleven a remediar una situación problemática causada por la empresa.

Crecimiento económico sostenible: el crecimiento económico sostenible en un mundo donde la desigualdad es extrema y la actividad económica puede causar daño irreversible al planeta. Las empresas cada día adquieren más conciencia al propender por un desarrollo sostenible que les garantice en el futuro poder contar con una fuente de recursos provenientes del medio ambiente, ya que ellos pueden contribuir a su conservación y hace viable su actividad.

Las tecnologías de la información y las comunicaciones: El avance en las tecnologías de la información y las comunicaciones (TIC) está presente en todos los niveles de la sociedad y ha permitido al hombre acceder fácilmente a todo tipo de información. Pese a que el interés por los factores que le aportan beneficios y valor material siguen delimitando el interés del consumidor (costos, calidad, diseño del producto/ servicio, etcétera), los consumidores de hoy, especialmente las nuevas generaciones, preferirán comprar a aquellas empresas que persigan una 


\section{La RSC en las empresas una estrategia rentable que necesita de impulsos legales}

Vol. 3, núm. 1., (2019)

Francisco Xavier Hugo Cárdenas; Cesar Eduardo Jiménez Rosero; Carlos Alberto Vasco Gualotuña; José Luis Saa Loor

conducta ética y asuman un compromiso claro con respecto a la responsabilidad social corporativa. Estos dos nuevos factores influyen potencialmente en la selección de determinadas marcas, productos y servicios que ofrecen las empresas que están comprometidas abiertamente con la sociedad y con el medio ambiente antes de preferir las marcas, los productos o los servicios de aquellas empresas que no aportan ningún tipo de beneficio para la sociedad. Hoy, el consumidor tiende a convertirse en un consumidor socialmente responsable.

Esquema de inclusión: Las estrategias de crecimiento empresarial determinadas desde el direccionamiento estratégico deben establecer un esquema de inclusión, que se comprometa en exaltar el valor del cliente, sin distinciones, eliminado la diferencia en la calidad del trato y la calidad del producto o servicio que se le ofrece. Este diseño debe enviar un mensaje positivo a los clientes y a la sociedad, con el objetivo de generar una fuerza impulsora para el crecimiento empresarial.

Agente de publicidad: El influjo de la Responsabilidad Social en el crecimiento empresarial invita a las empresas a reconsiderar los niveles de retribución que hacen a la sociedad y al medio ambiente y a contemplar la conveniencia de tener en sus planes estratégicos contemplado el tema de Responsabilidad Social como agente de publicidad; en éste, la empresa les plantea a sus grupos de interés y grupos especiales, a través de la publicidad, su gran sensibilidad hacia lo humano y lo ambiental.

La producción limpia: La empresa busca en la Responsabilidad Social dirigida hacia el crecimiento económico sostenible; para ello estimula la producción limpia, estableciendo políticas medioambientales en las compras, minimizando los residuos contaminantes y 


\section{La RSC en las empresas una estrategia rentable que necesita de impulsos \\ legales}

Vol. 3, núm. 1., (2019)

Francisco Xavier Hugo Cárdenas; Cesar Eduardo Jiménez Rosero; Carlos Alberto Vasco

Gualotuña; José Luis Saa Loor

estableciendo un sistema de reciclado que le permita generar el máximo aprovechamiento de las materias primas y la reducción de su costo.

La responsabilidad social interna: El crecimiento de la empresa se ve beneficiado al mejorar la confianza y el compromiso de los empleados, se perfecciona la motivación y se crea la cultura organizacional que propicie la generación de valor agregado en los productos o servicios, la creatividad y la innovación. La calidad de vida de los empleados de la organización debe reflejar el compromiso que tiene la empresa con prácticas responsables que los ayuden a aportar como individuos al mejoramiento de la sociedad.

De acuerdo con cada uno de los principios citados anteriormente, se puede decir que la responsabilidad social corporativa, debe ser hoy mirada como una estrategia rentable que posee condiciones viables para ofrecer a las empresas oportunidades de crecimiento económico sostenible, además les garantiza mantenerse en contacto directo con el medio circundante, razón, por la cual, se hace necesario construir un cuerpo jurídico que fije posiciones claras y determinadas a fin de que cada empresa, no pueda dejar a un lados su corresponsabilidad con la sociedad, todo lo contrario, introducir una transformación interna que se proyecte hacia lo externo como indicador de su propio crecimiento económico bajo nuevas visiones de desarrollo integral.

\section{Conclusiones.}

Cabe agregar que cada uno de los planteamientos citado a lo largo del recorrido interpretativo, conduce a concluir una serie de apreciaciones generales competentes que dan 


\section{La RSC en las empresas una estrategia rentable que necesita de impulsos legales}

Vol. 3, núm. 1., (2019)

Francisco Xavier Hugo Cárdenas; Cesar Eduardo Jiménez Rosero; Carlos Alberto Vasco Gualotuña; José Luis Saa Loor

cabida a entender la significación teórica que posee la responsabilidad social corporativa en el marco empresarial, para lo cual, es determinante generar estímulos mundiales que lleven a normarla como una herramienta que propicia el crecimiento económico sostenible de las empresas en las diferentes sociedades que estructuran el mundo actual. Entre ellas se citan las siguientes:

Como se mencionó anteriormente, cada vez más el avance en las tecnologías de la información y las comunicaciones, la curiosidad y alto nivel de formación de los clientes y su profundo compromiso con el medio ambiente hacen que éstos prefieran marcas, productos o servicios de las empresas que producen los mismos bienes y tienen una responsabilidad con la sociedad y el medio ambiente. Esta conciencia ambientalista de los clientes o consumidores busca compromisos por parte de las empresas con el medio ambiente; las empresas deben responder ofreciendo propuestas que equilibren los intereses de todos los grupos de interés, con el fin de implantar un crecimiento sostenible. En suma, el compromiso con la Responsabilidad Social arroja beneficios para los grupos de interés involucrados, ya que las empresas deben enfocar sus esfuerzos a generar beneficios que no se queden solo en el orden económico, sino que vayan mucho más allá, que transciendan al orden social.

Vale la pena mencionar que en aquellas empresas donde se incluye la Responsabilidad Social Corporativa dentro de sus planes estratégicos aumenta la rentabilidad; ésta no tiene por qué deteriorarse, por el contrario, debe existir una relación complementaria positiva entre la Responsabilidad Social y los niveles de rentabilidad en el mediano plazo. La sostenibilidad del sistema es prioritaria para todos los actores que participan en estas interrelaciones; la acción de 


\section{La RSC en las empresas una estrategia rentable que necesita de impulsos \\ legales}

Vol. 3, núm. 1., (2019)

Francisco Xavier Hugo Cárdenas; Cesar Eduardo Jiménez Rosero; Carlos Alberto Vasco

Gualotuña; José Luis Saa Loor

los gobiernos es insuficiente para lograr un balance sostenible entre lo económico, social y ambiental; por esta razón se hace indispensable que las empresas desarrollen una conciencia sobre la tarea crítica que tienen en estos frentes, y forma de cumplirla es beneficiando a todos sus grupos de interés.

También se debe tener en cuenta la capacidad de competencia que posee la empresa; pues, mediante ella logra obtener respuestas significativas en sus estructuras organizativas y los grupos de interés, además adelanta acciones esenciales para encaminar el proceso administrativo hacia la implementación de estrategias ampliamente efectivas que le permiten asegurar su desarrollo económico como patrón de crecimiento general. En otras palabras, se valora el entorno, las necesidades de los clientes, se hace apertura a nuevas acciones que buscan reducir los efectos colaterales como resultado a la presencia de integrar lo ético con lo social, para hacerlas cada día más socialmente competitivas.

\section{Bibliografía.}

Arias, F. (2012). El Proyecto de Investigación . Caracas : Episteme .

Baldillo, O. (2017). Rentabilidad y Productividad . Madrid: Paidós .

Bank, T. (2014). Responsabilidad Social . España : Narcea .

Blanco, T. (2014). Desarrollo Empresarial . México: Trillas .

Cortina, V. (2014). Responsabilidad Social y Política Empresarial . Chile: Vergara .

Ibañez, M. (2014). Estartegias Gerenciales para el Cambio. México: Continental.

Padilla, D. (2017). Inversión Rentable . Dinero , 66-82.

Sandoval, B. (2015). Esquema de Elaoración del Proyecto de Investigación . Caracas : Greco. 


\section{La RSC en las empresas una estrategia rentable que necesita de impulsos legales}

Vol. 3, núm. 1., (2019)

Francisco Xavier Hugo Cárdenas; Cesar Eduardo Jiménez Rosero; Carlos Alberto Vasco Gualotuña; José Luis Saa Loor

Ventura, S. (2018). El Proyecto de Investigación . Caracas: Oasis.

Villacis, C, Suárez, Y, y Guillin, X. (2016). Análisis de la Responsabilidad Social en Ecuador . Dialnet , 452-466.

Zavala, R. (2015). Responsabilidad Social Corporativa . México: Limusa. 